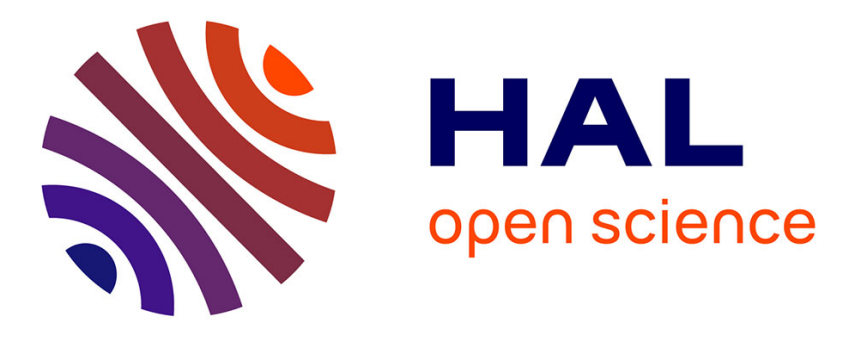

\title{
Dystrophin Dp71 is required for neurite outgrowth in PC12 cells.
}

\author{
Rosalinda Acosta, Cecilia Montañez, Lizeth Fuentes-Mera, Everardo \\ Gonzalez, Pablo Gómez, Leonor Quintero-Mora, Dominique Mornet, Luis M. \\ Alvarez-Salas, Bulmaro Cisneros
}

\section{To cite this version:}

Rosalinda Acosta, Cecilia Montañez, Lizeth Fuentes-Mera, Everardo Gonzalez, Pablo Gómez, et al.. Dystrophin Dp71 is required for neurite outgrowth in PC12 cells.. Experimental Cell Research, 2004, 296 (2), pp.265-75. 10.1016/j.yexcr.2004.01.015 . inserm-00149130

\section{HAL Id: inserm-00149130 https://www.hal.inserm.fr/inserm-00149130}

Submitted on 24 May 2007

HAL is a multi-disciplinary open access archive for the deposit and dissemination of scientific research documents, whether they are published or not. The documents may come from teaching and research institutions in France or abroad, or from public or private research centers.
L'archive ouverte pluridisciplinaire $\mathbf{H A L}$, est destinée au dépôt et à la diffusion de documents scientifiques de niveau recherche, publiés ou non, émanant des établissements d'enseignement et de recherche français ou étrangers, des laboratoires publics ou privés. 


\title{
Dystrophin Dp71 is required for neurite outgrowth in PC12 cells
}

\author{
Rosalinda Acosta, Cecilia Montanez, Lizeth Fuentes-Mera, Everardo Gonzalez, \\ Pablo Gomez , Leonor Quintero-Mora, Dominique Mornet, \\ Luis M.A lvarez-Salas, and Bulmaro Cisneros ${ }^{\mathrm{a}}$ *
}

Department of Genetics and Molecular Biology, Centro de Investigacion y de Estudios Avanzados del IPN, Avenida Instituto Politecnico Nacional 2508, Apartado Postal 14-740, C.P. 07000, Mexico D.F., Mexico

Institut de Biologie, Boulevard Henry IV, EA 701 Muscles et Pathologies Chroniques. 34060 Montpellier cedex 2, France

\begin{abstract}
:
To determine the role of Dp71 in neuronal cells, we generated PC12 cell lines in which Dp71 protein levels were controlled by stable transfection with either antisense or sense constructs. Cells expressing the antisense Dp71 RNA (antisense-Dp71 cells) contained reduced amounts of the two endogenous Dp71 isoforms. Antisense-Dp71 cells exhibited a marked suppression of neurite outgrowth upon the induction with NGF or dibutyryl cyclic AMP. Early responses to NGF-induced neuronal differentiation, such as the cessation of cell division and the activation of ERK1/2 proteins, were normal in the antisense-Dp71 cells. On contrary, the induction of MAP2, a late differentiation marker, was disturbed in these cells. Additionally, the deficiency of Dp71 correlated with an altered expression of the dystrophin-associated protein complex (DAPC) members a and $h$ dystrobrevins. Our results indicate that normal expression of Dp71 is essential for neurite outgrowth in PC12 cells and constitute the first direct evidence implicating Dp71 in a neuronal function. D 2004 Elsevier Inc. All rights reserved.
\end{abstract}

Keywords: PC12 cells; Dp71 function; Antisense RNA; Neuronal differentiation

* Corresponding author. Departamento de Genetica y Biologı Molecular, Centro de Investigacion y Estudios Avanzados del IPN. Av. I.P.N. 2508, Col, San Pedro Zacatenco, Mexico D.F., CP 07360, Mexico. Fax: +52-55-57-47-71. E-mail address: bcisnero@lambda.gene.cinvestav.mx (B. Cisneros). 


\section{Introduction:}

Duchenne muscular dystrophy (DMD) is an X-linked recessive disorder caused by mutations in the dystrophin gene [1]. Dystrophin is a 427-kDa rod-shaped protein consisting of four major domains $[1,14,25,26]$.The $\mathrm{N}$-terminal domain interacts with actin filaments, while the cysteine-rich and C-terminal domains associate with a complex of transmembrane glycoproteins and cytoplasmic proteins known collectively as the dystrophin-associated protein complex (DAPC) [16,41,44].

Analysis of dystrophin gene expression has led to the identification of several Cterminal dystrophin isoforms named according to their molecular weight $[9,10,15,20,31]$. A 70-to 75-kDa dystrophin protein (designed Dp71) has a unique seven residues $\mathrm{N}$ terminus fused to the cysteine-rich and C-terminal domains and is the most abundant dystrophin in a wide range of non-muscle tissues [5,17,28].In addition, Dp71 transcripts are alternatively spliced for exons 71-74 and 78 in several tissues; then multiple Dp71 protein products of $70-78 \mathrm{kDa}$ are generated [2,3]. Recently, we determined that alternative splicing regulates the cytoplasmic or nuclear localization of Dp71 $[19,34]$.

Although Dp71 is the major DMD gene product expressed in adult human brain tissue [21], its function is poorly understood. We have previously adopted the PC12 cell line as an in vitro neuronal model for studying Dp71 cellular function [12]. These cells express two Dp71 splicing isoforms; both of them spliced out for exon 71 and with exon 78 either present (Dp71d) or spliced out (Dp71f) [34]. Both Dp71 protein isoforms are upregulated during NGF-induced PC12 neuronal differentiation, Dp71f increases 9fold in total extracts, while Dp71d augments up to 7-fold in nuclear extracts; additionally, Dp71 isoforms showed distinct subcellular distributions from each other: Dp71f isoform

Table 1 Antibodies

Characteristics of the different antibodies used in this study

\begin{tabular}{|c|c|c|c|c|}
\hline Antibody & Position of antigen & Nature & Specificity & Reference/resource \\
\hline Dys2 & peptide 3669 - 3685 & Monoclonal & Dp71d isoform & Novacastra \\
\hline 2166 & C-ter 17 аа & Polyclonal & Dp71d isoform & {$[32]$} \\
\hline $5 \mathrm{~F} 3$ & alternative C-ter 31-aa & Monoclonal & Dp71f isoform & [17] \\
\hline JAF & C-ter 7 aа & Polyclonal & $\beta$-dystroglycan & [37] \\
\hline$\beta-521$ & peptide 521 - 738 & Polyclonal & $\beta$-dystrobrevin & {$[32]$} \\
\hline$\beta C T-F P$ & peptide 459 - 465 & Polyclonal & $\begin{array}{l}\beta \text {-dystrobrevin, } \\
\alpha \text {-dystrobrevin-1/2 }\end{array}$ & [32] \\
\hline MAP2 & fragment of MAP2 & Polyclonal & $\begin{array}{l}\text { microtubule-associated } \\
\text { protein } 2\end{array}$ & [29] \\
\hline p-ERK (E4) & Erk1pep P-Tyr-204 & Monoclonal & $\begin{array}{l}\text { phosphorylated } \\
\text { Erk1 and Erk2 }\end{array}$ & $\begin{array}{l}\text { Santa Cruz } \\
\text { Biotechnology, Inc. }\end{array}$ \\
\hline URD40 & $\begin{array}{l}\text { Rod domain of } \\
\text { mouse utrophin }\end{array}$ & Polyclonal & Utrophin and Up116 & {$[7]$} \\
\hline $\begin{array}{l}\gamma 2 \text { syntrophin } \\
\text { trk } \\
\text { actin }\end{array}$ & $\begin{array}{l}\text { P-Tyr-680/681 of TrkA } \\
\text { of human origin }\end{array}$ & $\begin{array}{l}\text { Polyclonal } \\
\text { Polyclonal } \\
\text { Monoclonal }\end{array}$ & $\begin{array}{l}\gamma 2 \text { syntrophin } \\
\text { phosphorylated TrkA, } \\
\text { TrkB and TrkC } \\
\text { all isoforms of actin }\end{array}$ & $\begin{array}{l}\text { Mornet, D. } \\
\text { Santa Cruz } \\
\text { Biotechnology, Inc. } \\
\text { Hermadez, M. }\end{array}$ \\
\hline
\end{tabular}


A

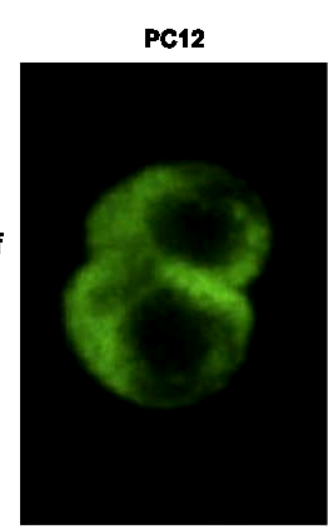

B

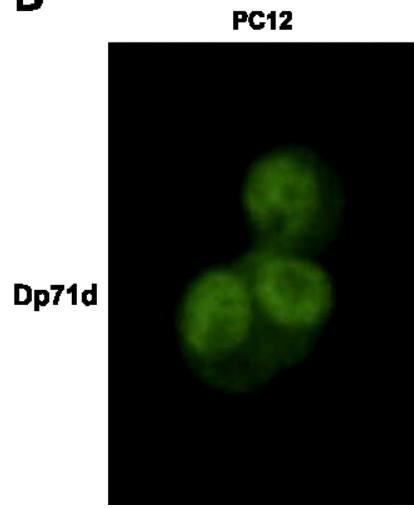

Control vector

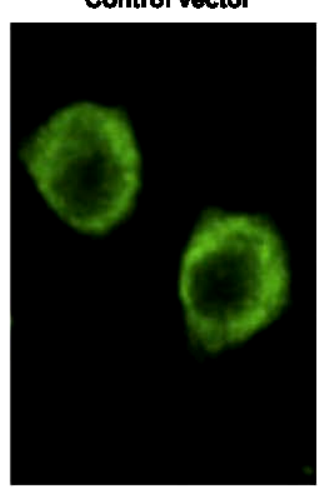

Antlsense-Dp71
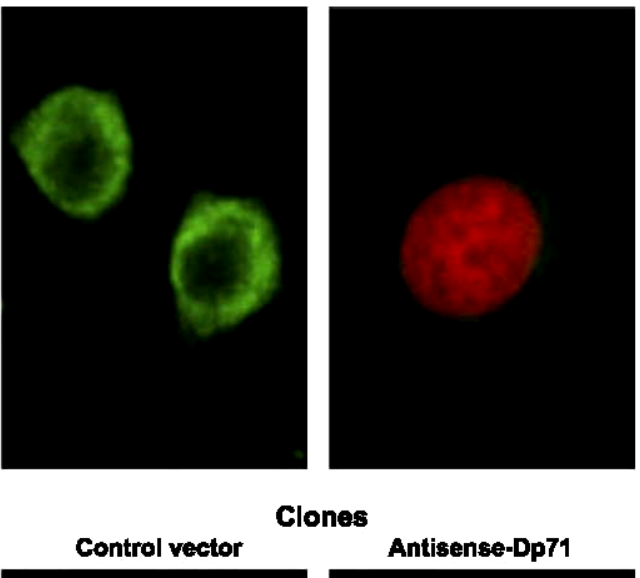

Clones

Antisense-Dp71

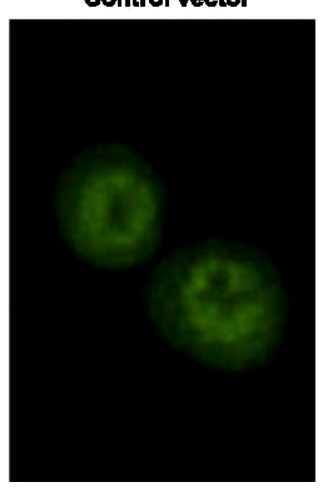

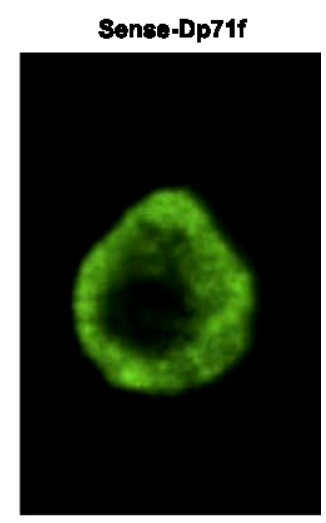

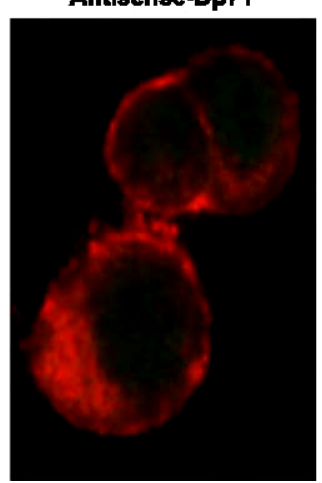

Sense-Dp71d

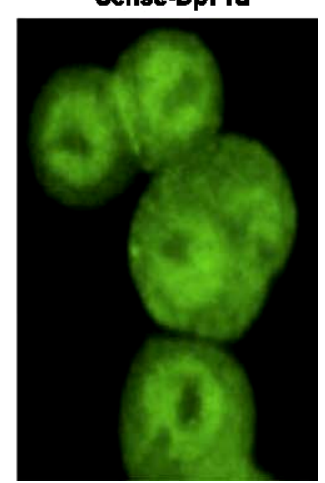

Fig. 1. Immunolocalization of Dp71 isoforms in PC12 cells stably transfected with Dp71 sense or antisense expression vectors. PC12 parental cells and the indicated stable clones were cultured on glass coverslips for confocal microscopy analysis. Cells were immunostained with monoclonal antibodies5F3 (A) and Dys2 (B) for the detection of Dp71f and Dp71d isoforms, respectively (green color). In (A), nuclei of antisense Dp71 cells, treated with antibody 5F3, were counter-stained with propidium iodine (red color). In (B), cytoplasmic actin of antisense-Dp71 cells, treated with antibody Dys2, was counter-stained with Texas red-phalloidin (red color). Dp71f staining was observed through the cytoplasm and cell periphery whereas that of Dp71d was concentrated in the nucleus. Note the immunostaining reduction of Dp71f and Dp71d protein isoforms in the antisense-Dp71 cells.

localizes in the cell body and neurites while Dp71d accumulates mainly in the nucleus [34]. These results suggest that each Dp71 variant may perform specific tasks in neuronal differentiation.

As an attempt to define the functional role that Dp71 plays in PC12 neuronal cells, we generated cell lines stably transfected with vectors expressing the Dp71 coding sequence in sense or antisense orientation. Antisense-Dp71 cells contain significantly less amount of Dp71f and Dp71d isoforms. The antisense-Dp71 cells showed impairment in their ability to display neurite outgrowth when induced to differentiate with NGF or dibutyryl cyclic AMP. The initial events in the neuronal differentiation pathway, such as the cessation of cell division and the activation of ERK1/2 proteins were unaltered in the antisense-Dp71 cells, however, the expression of the late marker MAP2 was disturbed. The deficiency in Dp71 protein levels had different effect on dystrophin-associated proteins expression; protein levels of $\beta$-dystroglycan, and $\gamma 2$-syntrophin remained unchanged, while those of $\alpha$ and $\beta$ dystrobrevins were disturbed. 


\section{Materials and methods}

\section{Plasmid constructions}

Expression vectors were prepared using human Dp71 cDNAs from pGFP-Dp71 plasmids [19]. The full-length (1.9 kb) Dp71 cDNA fragments, corresponding to Dp71f or Dp71d splicing isoforms were removed from the corresponding pGFP-Dp71 vectors with EcoRI and ligated into the same restriction enzyme site in pDGFP. After plasmid amplification, the orientation of Dp71 fragments was ascertained by restriction enzyme analysis. Hence, vectors generating sense (pDP71f and pDp71d) or antisense (pDp71As) Dp71 RNA were obtained. An additional vector expressing in antisense orientation the first 392 bp from 5Vend of the Dp71 cDNA was constructed by digestion of pDp71As with BamHI and further vector ligation. Expression vector pDGFP, which contains the early CMV promoter and the neomycin resistance gene was constructed by deleting the GFP coding sequence from pQBI25 plasmid (Quantum Biotechnologies) with SacII and ClaI digestion, filling the restricted enzyme sites with T4 DNA polymerase and recircularizing with T4 DNA ligase. Plasmid pDGFP with no Dp71 insert was used as control vector.

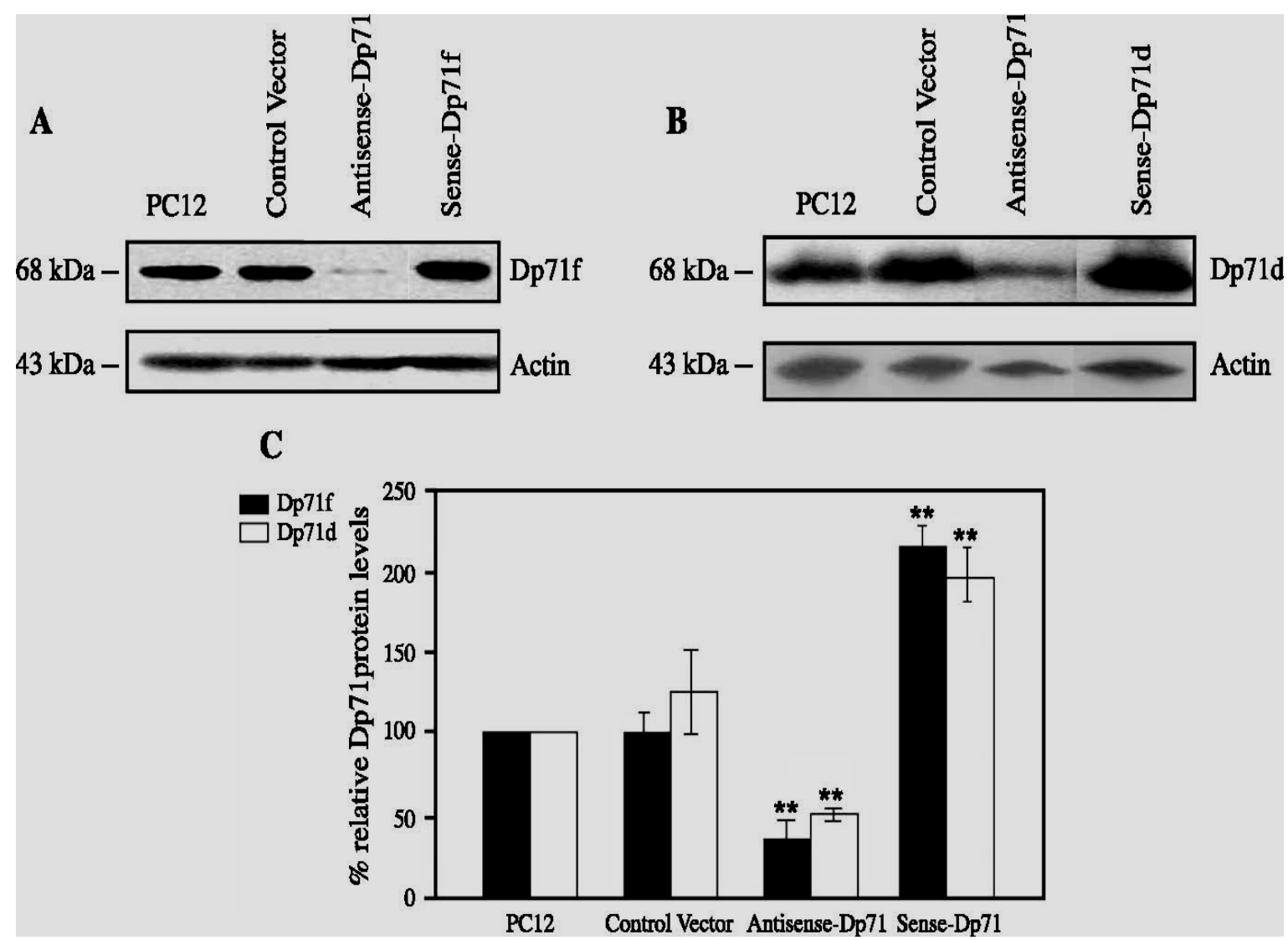

Fig. 2. Expression of Dp71 protein isoforms in the stable PC12 clones. Protein extracts obtained from PC12 parental and the indicated stably transfected PC12 clones were subjected to immunoblot analysis. (A) Immunodetection of Dp71f isoform was carried out with the monoclonal antibody 5F3. (B) Immunodetection of Dp71d isoform was performed using the rabbit antisera 2166. Membranes were stripped and reprobed with monoclonal antibody anti-actin. Migrations of Dp71 isoforms and actin are indicated at the right. Positions of protein marker are shown at the left. (C) Protein levels of Dp71 isoforms were normalized with respect to actin protein levels. Three different clones from each group of stable cell lines (control-vector, antisense-Dp71, sense-Dp71f, and sense-Dp71d) were analyzed separately in duplicate experiments, and the average value F SD obtained from each one is presented in the graph. Asterisks denote significant differences $(\mathrm{P}<0.05)$ from control-vector cells. Antisense-Dp71 cells exhibited a significant decrease in the expression of Dp71d and Dp71f isoforms. 


\section{Cell culture and generation of stable transfectans}

PC12 cells were grown in RPMI-1640 medium supplemented with $10 \%$ horse serum, 5\% fetal bovine serum, $100 \mathrm{U} / \mathrm{ml}$ penicillin and $0.1 \mathrm{mg} / \mathrm{ml}$ streptomycin, and maintained at 37jC in a humidified incubator with a 5\% CO2 atmosphere. For stable transfection, PC12 cells were grown to $60 \%$ confluence onto collagen-coated $100-\mathrm{mm}$ dishes, transfected with $5 \mathrm{Ag}$ of plasmid DNA pre-mixed with 5 AlofPlusreagent and8 Al of lipofectamine (Gibco) in serum-free Opti-MEM medium (Gibco). After a 5-h incubation period, the transfection mixture was removed by washing with Opti-MEM and cells were maintained in RPMI-1640 supplemented with sera. Selection of stable transfectans was initiated $48 \mathrm{~h}$ after transfection with $500 \mathrm{Ag} / \mathrm{ml}$ of G418 (Invitrogen), a neomycin analog.

\section{Cell differentiation assay}

To induce differentiation, $2 \times 10^{4}$ cells were seed onto collagen-coated 6-well plates, and treated with50 ng/ml2.5S nerve growth factor (NGF, Gibco) or with $1 \mathrm{mM}$ dibutyryl cyclic AMP (Sigma). Medium containing NGF or dibutyryl cyclic AMP was changed every third day. To determine neurite outgrowth, the number of neurites per cell and the relative length of the neurite, in designated microscopic fields, were measured at 6 days of differentiation. The differentiation score was defined as follows: $0=$ no change (round or polygonal cells), 1 =any extension of one or more neurites, each less than one body length, 2 = extension of one or more neurite between one and two cell body length, $3=$ at least one neurite of more than two body length in size $[4,13]$.

\section{A}

Control Vector

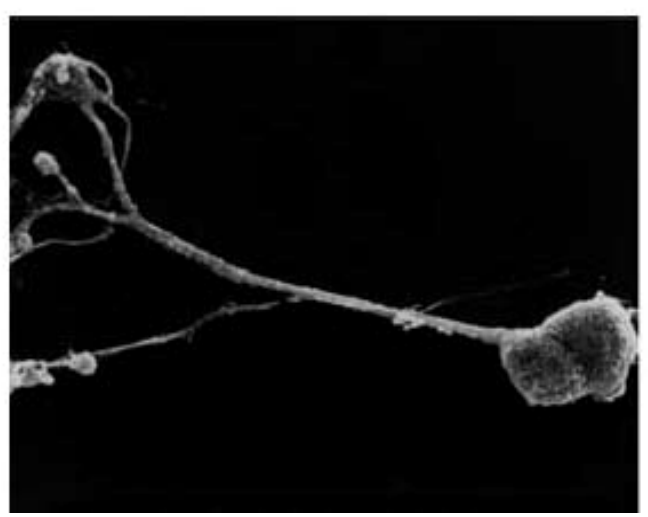

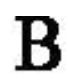

\section{Antisense-Dp71}

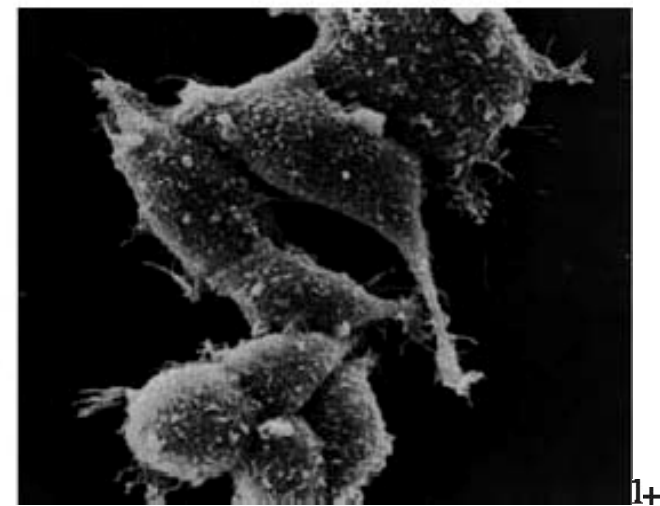

Fig. 4. Scanning electron microscopic analysis of control-vector and antisense-Dp71 clones. After6 days of NGF treatment, control-vector cells present many large neurites (A), while antisense-Dp71 cells displayed only very short stubby processes (B). 


\section{Cell proliferation assay}

$2 \times 10^{4}$ densities. Cell number was determined by direct counting of the cells at various

Untreated and NGF-treated cells were plated onto collagen-coated 6 well plates at times using a Neubauer chamber.

\section{Immunofluorescence assays}

For single immunofluorescence staining, cells plated onto poly-D-lysine-coated glass coverslips were fixed and permeated for $5 \mathrm{~min}$ with 3\% paraformaldehyde $/ 0.5 \%$ tritonX100 in Cytoskeleton Buffer (10 mM MES, $150 \mathrm{mM} \mathrm{NaCl,} 5$ mM EGTA, 5 mM $\mathrm{MgCl} 2,5 \mathrm{mM}$ glucose) and then incubated $15 \mathrm{~min}$ with 3\% paraformaldehyde.

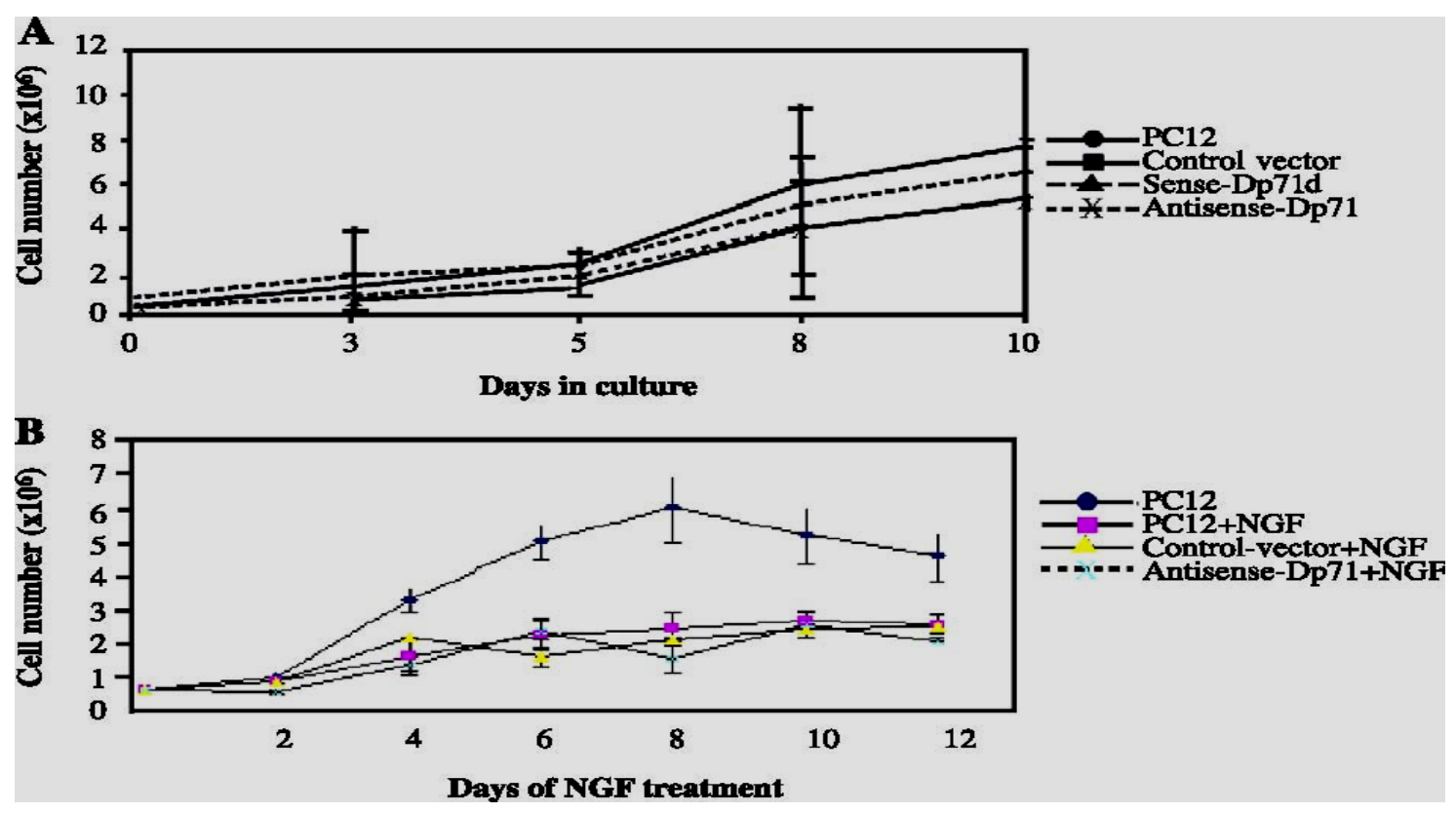

Fig. 3. Growth rates of undifferentiated and NGF-treated PC12 stable clones. Cultures of parental PC12 cells and stably transfected clones were plated onto collagen-coated 24-well plates at density of8x $10^{5} /$ dish without NGF (A) or at density of $3 \times 10^{3}$ cells/dish with NGF (B). The cell numbers were determined by direct counting at the indicated days, as described in Material and methods. Three different clones from each group of stable cell lines (control-vector, antisense-Dp71, and sense-Dp71f) were analyzed separately in duplicate experiments, and the mean value obtained from each one is presented in the graph. In the absence of NGF, the cellular growth of the stably transfected clones was not significantly different from that of the parental PC12 clone (A). NGF-treated cultures from stably transfected and parental clones stopped dividing since1day after NGF addition, while untreated parental PC12 cells continued growing (B).

Fixed cells were blocked 20 min with $0.5 \%$ gelatin and incubated overnight at $4 \mathrm{jC}$ with primary antibodies (Table 1). The day after, coverslips were incubated in PBS for $1 \mathrm{~h}$ with a fluorescein-conjugated secondary anti-mouse antibody. For counter staining, cells were incubated for 20 min at room temperature with Texas Red-phalloidin (Molecular Probes) or during 5 min with Propidium iodine (Sigma). After washing once with PBS, coverslips were mounted with VectaShield (Vector Laboratories Inc) and observed in an 
epifluorescence Nikon microscope coupled with a confocal system BIO-RAD MRC600. From each image, 9 to 12 optical Z-sections (0.2-0.5 Am thick) were scanned using the laser confocal microscope dual channel imaging system.

\section{Immunoblotting assays}

Cells were centrifuged and resuspended in complete protease inhibitor cocktail (Boehringer) containing $50 \mathrm{mM}$ Tris- $\mathrm{HCl}$ (pH 8.0) and 0.1\% DNase1. Homogenates were sonicated and protein concentrations were determined by the Bradford method. Aliquots (40 Ag) were resolved on denaturing 3- 10\% gradient SDS-PAGE and electrotransferred to nitrocellulose membranes. Immunoblots were probed with either monoclonal antibodies or rabbit antisera (Table 1), and were developed using the ECL Plus kit (Amersham BioSciences). Quantitative analysis of immunoblots was performed on a digital Science ID system (Kodak).

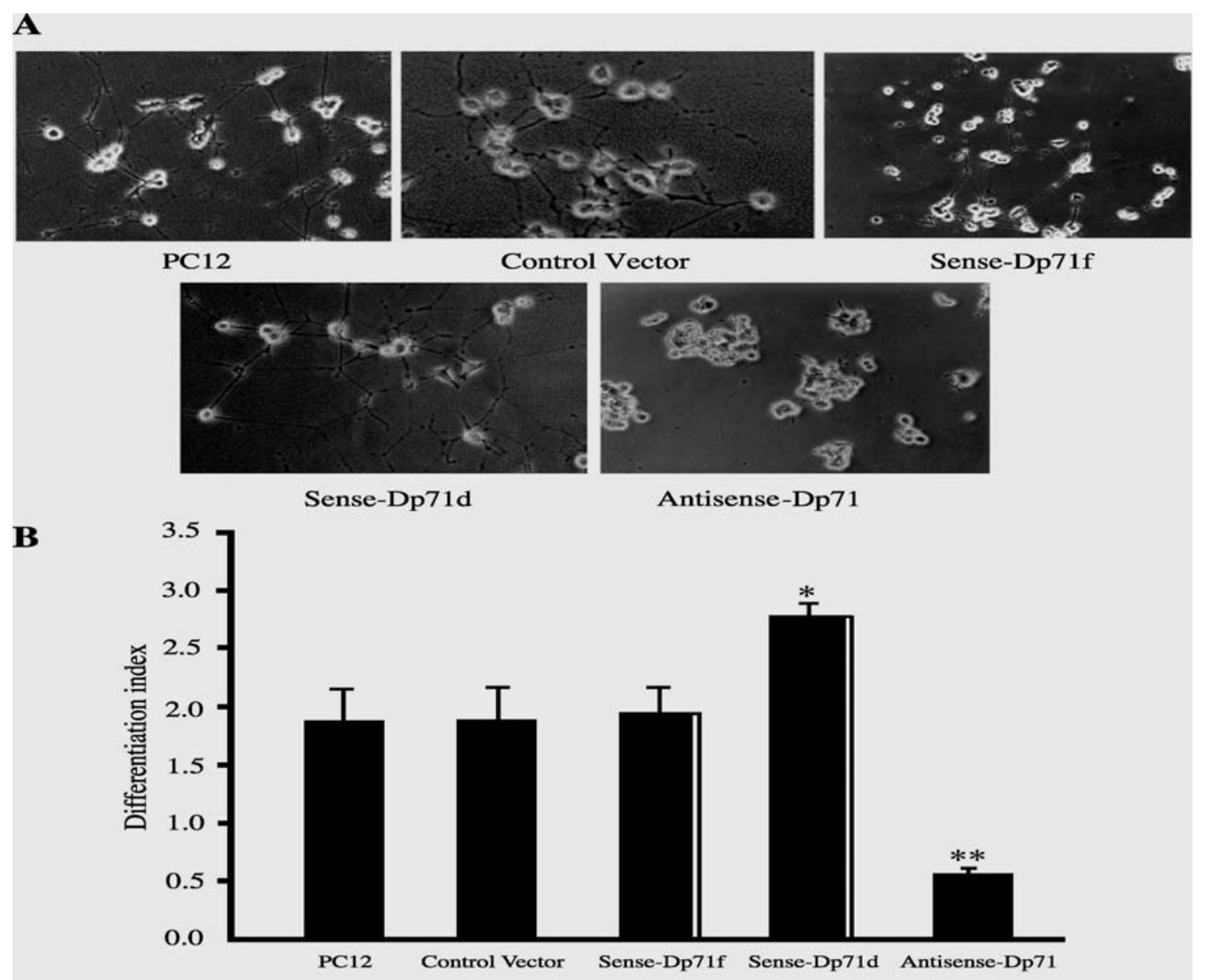

Fig. 5. Effect of Dp71 antisense treatment on NGF-induced neuronal differentiation of PC12 cells. Parental and stably transfected PC12 clones were grown on collagen-coated dishes in the presence of NGF. (A) Phase contrast micrographs illustrating typical cell morphologies of the indicated cultures were obtained at day 6 of NGF exposure. (B) Differentiation scores were calculated as described in Material and methods. Five different clones from each group of stable cell lines (control-vector, antisense-Dp71, sense-Dp71f, and sense-Dp71d) were analyzed separately in duplicate experiments, and the mean value F SD obtained from each one is shown in the graph. Asterisks denote significant differences $(P<0.05)$. Neurite outgrowth 


\section{Scanning electron microscopy}

Cell samples were fixed in 2.5\% glutaraldehyde, dehydrated in ethanol, and dried at critical point with CO2 (maximum temperature 39jC; maximum pressure $110 \mathrm{psi}$ ) in a SAMDRY-780 dryer (Tousimis Research, Rockville, MD). The cells were then covered with gold ions in a JEOL JFC-110 ion sputtering (Tokyo, Japan) and analyzed in a JEOL 35-C scanning electron microscope.

\section{Statistics}

Statistical analyses were performed using a two-tailed Student's t test.

\section{Results}

\section{Establishment and characterization of stable PC12 clones}

To begin our assessment of Dp71 protein function in rat PC12 cells, we established stable clones by transfecting PC12 cells with derivative pQBI25 vectors that contained no Dp71 insert (control-vector clones) or either the full-length Dp71 cDNA or the first $392 \mathrm{bp}$ from its 5V-end in antisense orientation relative to the CMV promoter (antisense-Dp71 clones). For comparison, we also created stable clones overexpressing each of the two Dp71 isoforms that are naturally expressed in PC12 cells; a variant lacking the amino acids encoded by exons 71 and 78 (sense-Dp71f clones) and an isoform lacking amino acids encoded by exon 71 but preserving those encoded by exon 78 (sense-Dp71d clones). Stable clones were maintained in culture for about 3 months and for each construct a total of five, randomly chosen, isolated colonies were selected to continue their characterization. Stably transfected cell lines were characterized by resistance gene expression, genomic integration of the DNA vector and expression of exogenous sense or antisense Dp71 RNA (data not shown). Given that similar phenotypic responses were observed between the stable clones expressing in antisense orientation the full-length cDNA or the first 5V-end $392 \mathrm{bp}$, representative images or an average of the data obtained from the different antisense clones analyzed are shown herein.

\section{Antisense-Dp71 cells show decreased Dp71 protein expression}

The effectiveness of the antisense-Dp71 constructs at reducing Dp71 concentrations in the antisense-Dp71 cells was evaluated by immunofluorescence confocal microscopy and Western blot analyses. To differentiate between Dp71 isoforms, several antibodies raised against the C-terminal domain of Dp71 were utilized; Dys2 and 2166 that recognize Dp71d, and 5F3 for the detection of Dp71f (Table 1). In parental and controlvector cells, immunostaining of Dp71f revealed a scattered signal throughout the cytoplasm, being stronger in the cell periphery (Fig. 1A) whereas the staining of Dp71d accumulated in the nucleus and to lesser extension in the cytoplasm (Fig. 1B), as previously reported [34].In the antisense-Dp71 cells, cytoplasmic staining of Dp71f and 
the nuclear signal of Dp71d were dramatically reduced (Figs. 1A and B). On the other hand, the stronger staining of Dp71d and Dp71f observed in the sense-Dp71d and Dp71f cell lines, respectively, confirmed the exogenous overexpression of these isoforms (Figs. $1 \mathrm{~A}$ and $\mathrm{B})$.

A typical immunodetection of the Dp71f isoform using the 5F3 antibody is shown in Fig. 2A. A band with an apparent molecular mass of $70 \mathrm{kDa}$ was observed in the protein extracts of untrasfected and control-vector cells. The intensity of this band clearly increased in the senseDp71f cells, confirming its specificity. On contrary, Dp71f immunoreactivity was practically absent in the antisense-Dp71 clones. By using the antibody 2166, a single band at the 68-kDa marker, which must correspond to Dp71d, was observed in the parental and control-vector cells (Fig. 2B). As expected, Dp71d immunoreactivity was strongly diminished in the antisense-Dp71 clones and

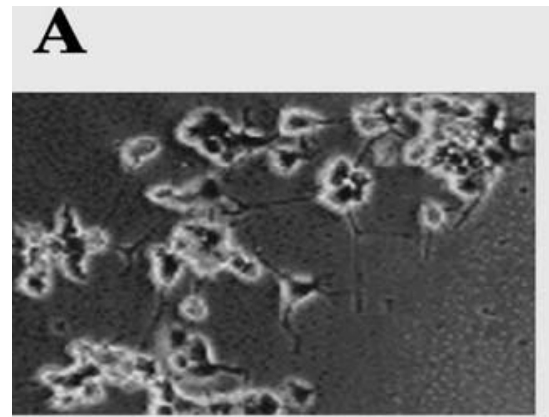

PC12

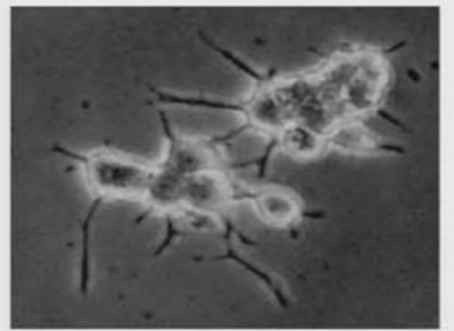

Control vector

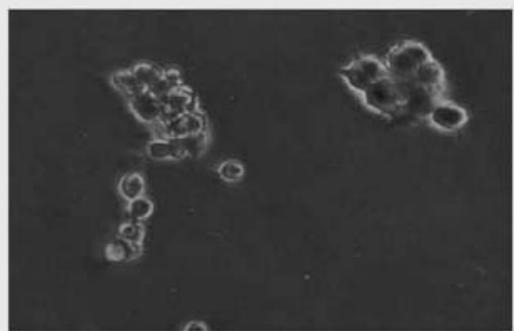

Antisense-Dp71

\section{B}

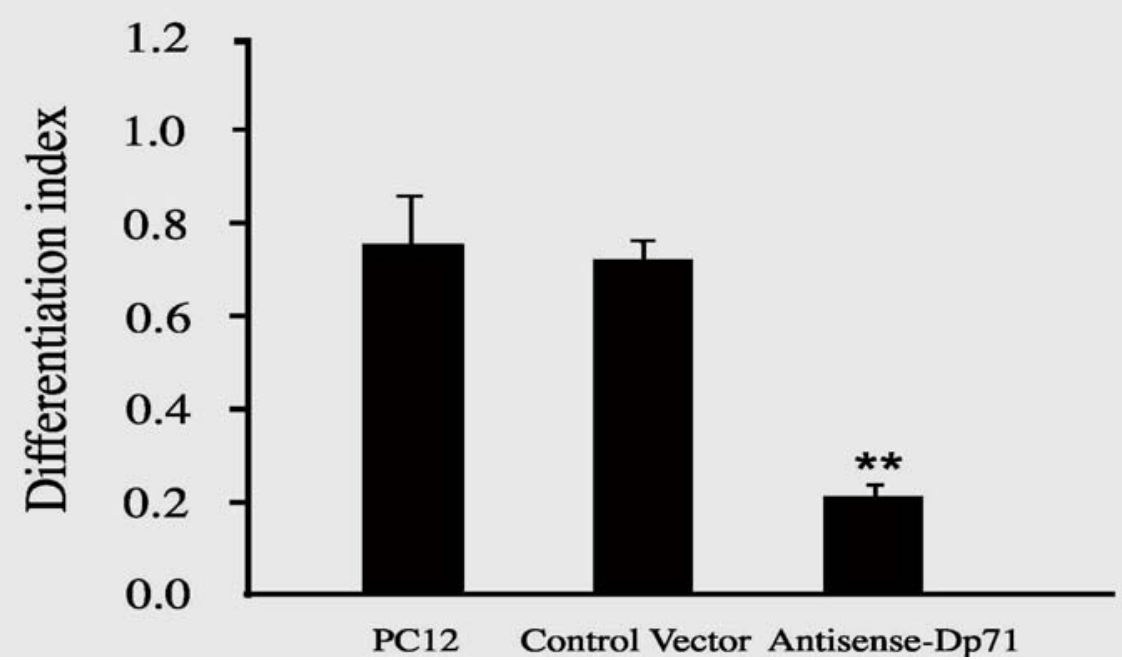

- ItrFig. 6. Effect of Dp71 antisense treatment on neurite outgrowth in dibutyryl cyclic AMP-treated stable clones. (A) Phase contrast micrographs illustrating typical cell morphologies of the indicated cell lines were obtained at day 6 of dibutyryl cyclic AMP exposure. (B) Differentiation scores were calculated as described in Material and methods. Five different clones from each group of stable cell lines (control-vector and antisenseDp71) were analyzed separately in duplicate experiments, and the average value F SD obtained from each one is shown in the graph. Asterisks denote significant differences $(\mathrm{P}<0.05)$. Neurite outgrowth is inhibited in the dibutyryl cyclic AMP-treated antisense-Dp71 cells. 
substantially increased in the sense-Dp71d cells. Fig. 2C shows the quantitative analysis of Dp71 protein isoforms expression in the stable clones; antisense-Dp71 clones exhibited an average reduction of $70 \%$ and $48 \%$ in the protein levels of Dp71f and Dp71d, respectively, while sense-Dp71f and -Dp71d clones presented a 2-fold average increase in the protein expression of the respective overexpressed Dp71 isoform.

\section{Cellular proliferation is not affected by the altered expression of Dp71 protein isoforms}

To ascertain whether the perturbation in Dp71 protein expression affects cellular growth, the proliferation rates of the different stably transfected clones were quantified during a time period of 10 days. Fig. 3A shows that the rates at which the antisense or sense-Dp71 clones proliferate were not significantly different than those of the parental or control vector-transfected clones.

\section{Decreased expression of Dp71 disturbs neurite outgrowth of PC12 cells}

To determine whether diminished levels of Dp71 affect neuronal phenotype of PC12 cells, we examined the morphology of stable clones at light (LM) and electron microscopic (EM) levels. In the absence of NGF, the parental PC12 cells as well as all the stably transfected clones showed normal growth under the LM analysis, characterized by the presence of rounded cells growing in small clumps (data not shown). After 6 days of NGF treatment, we found by the LM and EM analyzes that the antisense-Dp71 cells did not respond to NGF with the features that accompanied the neuronal differentiation of parental PC12 cells, such as flattening, increase in cell body, and outgrowth of neurites (Figs. 4A and 5A); instead, these cells exhibited only very short stubby processes (Figs. 4B and 5A), which failed to elongate even with longer NGF exposures (data not shown). Interestingly, the cell line overexpressing the Dp71d isoform showed an enhanced response to NGF, the length, density, and branching patterns of its processes appeared to be greater than those observed in parental PC12 cells (Fig. 5A). Measurement of neurite outgrowth confirmed these observations; relative to untransfected cells, an average reduction of $69 \%$ in the differentiation score was observed in the antisense-Dp71 clones whereas sense-Dp71d cell lines exhibited an average increase of $49 \%$ in this parameter (Fig. 5B). Control-vector and sense-Dp71f stably transfected clones displayed differentiated morphologies (Fig. 5A) with differentiation scores that were statistically similar to that found in parental cells (Fig. 5B).

To evaluate the extent of the defect in differentiation observed in the antisense-Dp71 clones, we analyzed the response of these cells to dibutyryl cyclic AMP, an agent that also induces a neurite outgrowth signaling pathway [23,43]. After 6 days of treatment, we observed that antisense-Dp71 clones displayed an absence of neuritic processes (Fig. 6A), rendering an average reduction of 75\% in their differentiation score (Fig. 6B). 
$\mathbf{A}$
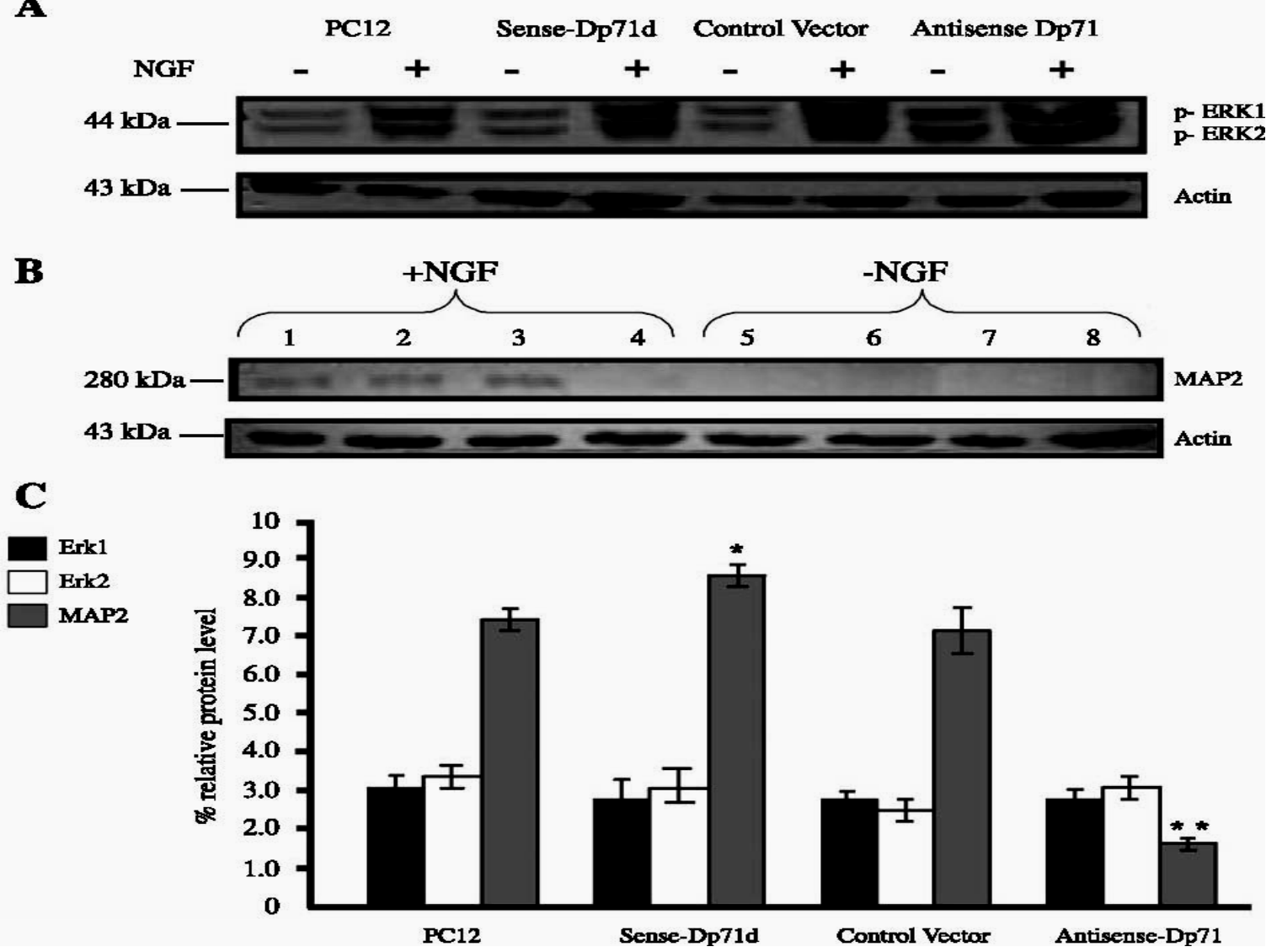

Fig. 7. Expression of MAP2, a late differentiation marker, is disrupted in the antisense-Dp71 cells. Western blot analyses of parental PC12 cells and stable transfectans showing the abundance of phospho ERK1/2 and MAP2 proteins before (-) and after (+) NGF induction. Cell cultures were exposed to NGF during40minforthe detection of phospho ERK1/2(A),or7days for the detection of MAP2(B). Lanes:1and5, PC12 cells;2and6, control-vector cells;3 and7, sense-Dp71d cells;4and8, antisense-Dp71 cells.(C) Relative protein levels are expressed as fold increase over protein values of untreated cells at time 0min. Anti-actin monoclonal antibody was used to normalize the amount of protein input in the gels. Three different clones from each group of stable cell lines (control-vector, antisense-Dp71, and sense-Dp71d) were analyzed separately in duplicate experiments, and the mean value F SD obtained from each group is shown in the graph. Asterisks denote significant differences $(\mathrm{P}<0.05)$. The activation of ERK1/2 proteins was unaltered in the antisense-Dp71 cells while the expression of MAP2 was disturbed.

\section{Analysis of the NGF-induced neuronal differentiation pathway in the antisense-Dp71 cells}

To identify events in the PC12 neuronal differentiation pathway that could be affected in the antisense-Dp71 cells, we undertook an analysis of molecular markers of early and late stages of NGF-induced PC12 neuronal differentiation. The activation of the two closely related MAP kinases (ERK1/ERK2), detected by their phosphorylation, occurred at statistically similar rate between the antisense-Dp71 clones and the controlvector cell lines (Figs. 7A and C). Another of the immediate responses of PC12 cells to NGF treatment is the cessation of cell division to begin differentiation program. Hence, we 
$\mathbf{A}$

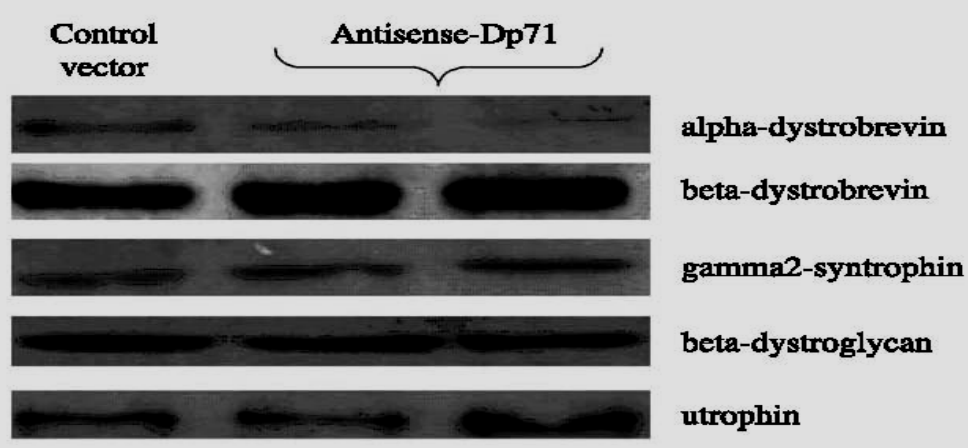

B

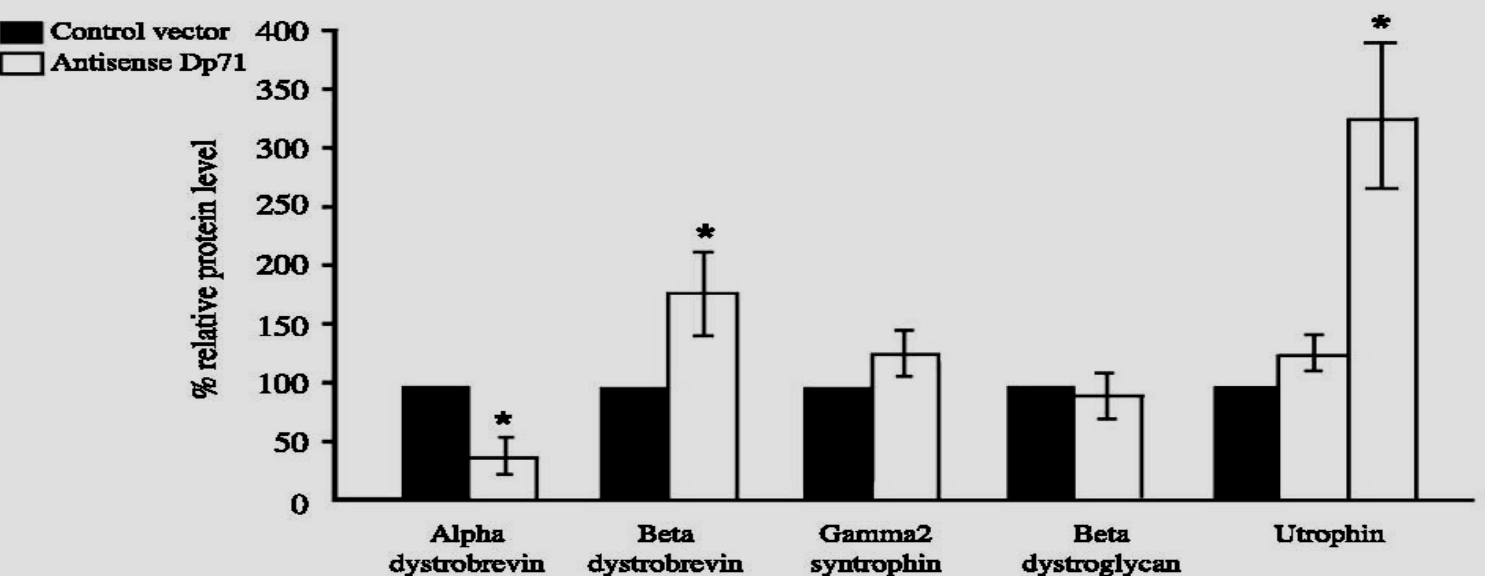

Fig. 8. Expression of DAPC in the antisense-Dp71 cells. (A)Total protein extracts from control-vector and antisense-Dp71 cell lines were subjected to Western blot analyses using specific antibodies against a and h dystrobrevins, $\gamma 2$-syntrophin, h-dystroglycan, and utrophin. Membranes were stripped and reprobed with monoclonal antibody anti-actin. (B) Three different clones from each group of stable cell lines (controlvector and antisense-Dp71) were analyzed separately in duplicate experiments, and the average value $+/-$ SD of the protein levels obtained from each group is shown in the graph. Since two different utrophin expression patterns were observed among antisense-Dp71 clones, a representative clone from each expression pattern was selected to be shown. Asterisks denote significant differences $(\mathrm{P}<0.05)$ from control-vector cell line. Antisense-Dp71cells exhibited an altered expression of $\alpha$ and $\beta$ dystrobrevins, while that of $\beta$-dystroglycan and $\gamma 2$-syntrophin remained unchanged. In addition, elevated utrophin protein levels were found in one of the antisense-Dp71 clones analyzed (panels A and B).

quantified the proliferation rate of antisense-Dp71 and control cultures after NGFinduction. Fig. 3B shows that all the stably transfected clones stopped dividing since 1 day after NGF addition and displayed close similar growth rates. Untreated parental PC12 cells continued growing with a doubling time of $62 \mathrm{~h}$. Regarding the expression of the late differentiation marker MAP2, the control cultures as well as the sense-Dp71d clones displayed a substantial 7-to 9-fold increase in the average levels of this protein after NGF treatment; in contrast, its expression appeared to be clearly disturbed in the antisense Dp71 cells, as judged by its modest response to NGF induction, 1.5-fold increase (Figs. 7B and C).

\section{Expression of the DAPC in the antisense-Dp71 cells}

To determine whether the perturbation in Dp71 expression alters the stability of the dystrophin-associated protein complex (DAPC), total protein levels of DAPC's members were analyzed from control and antisense-Dp71 cultures (Fig. 8). In the antisense-Dp71 clones, the average expression of h-dystroglycan, and g2-syntrophin was similar to that observed in control-vector transfected clones (panels A and B). On contrary, protein 
expression of dystrobrevins was altered in the antisense cell lines: whereas protein levels of a-dystrobrevin were $70 \%$ diminished those of h-dystrobrevin exhibited an average increase of 75\% (panels A and B). We also examined utrophin expression in the antisenseDp71 clones, a dystrophin homologue expressed in PC12 cells [39]. Compared with control culture, we found significantly higher utrophin levels in one of the antisense-Dp71 clones analyzed (panels A and B).

\section{Discussion}

We generated stably transfected PC12 cell lines that express either reduced or increased levels of Dp71d and Dp71f isoforms. Deficiency in Dp71 protein levels resulted in an impairment in the ability of PC12 cells to extend neuritis when induced to differentiate with NGF or dibutyryl cyclic AMP. Given that all antisense-Dp71 clones exhibited similar phenotypic response and that none of the clones expressing the control vector showed this alteration, it seems unlikely that our results were attributable to selecting G418-resistant PC12 clones that were simply defective in neurite outgrowth.

To begin to understand the mechanism by which the deficiency of Dp71 obstructs PC12 neuritogenesis, we tried to identify events in the differentiation pathways that could be affected. On the one hand, it is well known that NGF activates receptor tyrosine kinases thereby initiating a cascade of events involving activation of the small GT-binding protein ras, which stimulates a kinase cascade consisting of Raf-1, B-raf, MAP kinase, MEK, and extracellular-signalregulated kinases (ERK1 and ERK2), whose sustained activation by NGF is sufficient for neuritogenesis in PC12 cells $[18,24]$. The phosphorylated ERK1/2 translocates to the nucleus and activates new gene expression leading to neurite outgrowth [24]. On the other hand, dibutyryl cyclic AMP activates protein kinase A (PKA) which also leads to neurite outgrowth.

Immediate responses to NGF treatment, such as cessation of cell division and induction of ERK1/2, are unaltered in the antisense-Dp71 cells. On contrary, NGF-induced expression of MAP2, a late marker of PC12 neuronal differentiation [8] was markedly inhibited in the antisense cells. Taken together, our results strongly suggest that native Dp71 is required for late stages of NGF-induced PC12 neuronal differentiation, wherein the hallmark is the outgrowth of neurites. Supporting this interpretation, we have observed that endogenous isoforms of Dp71 augment their levels in PC12 cells over a time course of days after NGF treatment [34], an expression pattern characteristic of genes associated with the acquisition of neuronal phenotype [24].

With respect to the role of Dp71 on dibutyryl cyclic AMP-induced neuronal differentiation, since this pathway is quite less characterized than NGF signaling, it is hard to envisage at what step this protein participates. In fact, a lasting discussion has taken place in the literature regarding the potential relationship between the differentiation mechanisms of these two agents. Early reports established clear differences between the responses of PC12 cells to NGF and cyclic AMP; the latter causes a rapid, but unstable reorganization of the cytoskeleton by a RNA synthesis-independent event, while NGF achieves a more stable cytoskeleton reorganization by an RNA synthesis-dependent mechanisms $[23,43]$. In addition, it has also observed that cyclic AMP and NGF have complementary effects on these cells, together producing an enhanced response over that with either agent alone [23]. These results suggest that cyclic AMP and NGF can act to initiate neurite outgrowth by different mechanisms. However, an activation of ERK kinases by cyclic AMP/PKA signaling through a Braf/Raf1-dependent pathway has been reported [42], which indicates that both differentiation routes may converge. Supporting this hypothesis, it has been observed in primary neurons that cyclic AMP/PKA-mediated signaling and ERK pathway act independently at the initiation of neuritogenesis but 
become coupled during later stages of neuronal development [43]. Therefore, the question of distinct versus common final signaling pathways remains open.

At the present stage, it is difficult to establish a role for each Dp71 isoform in neuritogenesis. On the one hand, since a retrograde actin flow is generated by actin polymerization and myosin motors during neurite outgrowth [36,38], it is possible that Dp71f, the predominant cytoplasmic isoform, may modulate the architectural changes occurring in the cytoskeletal network during the NGF or cyclic AMP signaling, through its direct interaction with both actin [27] and h-dystroglycan [11]. On the other hand, several findings suggest that Dp71d, the predominant nuclear isoform, is also implicated in neurite outgrowth. First, transfected clones overexpressing Dp71d isoform exhibited an enhanced extension of neurites, while those overexpressing Dp71f variant did not. Second, we have observed that nuclear accumulation of Dp71d occurred during the late stages of PC12 cell differentiation when development of neurites is evident [34]. Finally, we have found Dp71d associated with the PC12 nuclear matrix (unpublished results), a subnuclear structure involved in RNA processing and chromatin remodeling [33]. Therefore, it is temping to speculate that Dp71d may mediate nuclear responses, which in turn account for the ability of this isoform to participate in the regulation of differentiation signaling. Currently, antisense strategies directed to reduce specifically the expression of each Dp71 isoform are underway in our laboratory.

It is has been observed that Dp71 is necessary for the formation and/or stabilization of the DAPC in brain [22]. We showed here that decreased expression of Dp71 in the antisense-Dp71cells correlated with altered protein levels of dystrobrevins, while protein expression of h-dystrobrevin increased that of $\alpha$-dystrobrevin showed a reduction. These results suggest that two alternative dystrobrevin-containing DAPC are present in PC12 cells, as previously observed in several tissues [6,32], and that Dp71 is necessary for the stability of the a-dystrobrevin-containing DAPC. Consistent with this interpretation, we have found by immunofluorescence assays that Dp71 and a-dystrobrevin distribute together in the cytoplasm and nucleus of PC12 cells (unpublished results). Further experiments are necessary to ascertain if the altered expression of dystrobrevins takes part in the neurite outgrowth impairment displayed by the antisense-Dp71 cells. The protein expression of h-dystroglycan and g2-syntrophin was unaltered in the Dp71-antisense cells suggesting that besides Dp71, other proteins, such as utrophin, are able to stabilize the DAPCs in PC12 cells.

Overall, our results indicate that native Dp71 is essential for neurite outgrowth in PC12 cells and to our knowledge constitute the first direct evidence indicating a role for Dp71 in neuronal function and development. Supporting our results, it has been observed that Dp71 protein expression increased in parallel with brain development [30]. Furthermore, C-terminal mutations in dystrophin, which would adversely affect Dp71 expression, are associated with mental retardation [35], indicating that Dp71 plays a role in central nervous system development. Nevertheless, preliminary examination on Dp71-null mice has not yet detected conspicuous morphological or histological abnormalities in central nervous system development when compared with wild type mice [40]. A possible explanation for this discrepancy is that in Dp71 null mice, the lack of Dp71 is at least partially compensated for by another product of the DMD gene or by a dystrophin-related protein (i.e., utrophin), whereas in the Dp71-antisense cells, such possibility seems unlike because we have previously established that PC12 cells, besides Dp71, do not express any other DMD gene product $[34,39]$. Furthermore, a functional compensation by utrophin appears unlikely too because all antisenseDp71 clones displayed very similar phenotypic 
response; however, we observed elevated utrophin levels in only one of the antisenseDp71 clones analyzed. Hence, it appears that elevated utrophin expression is related to the genetic background of a particular clone rather than to a compensatory mechanism for the deficiency of Dp71.

\section{Acknowledgments}

We would like to thank Dereck Blake for supplying antibodies 2166, URD40, h-521 and h-CT-FP and Dr. Manuel Hernandez for supplying an anti-actin monoclonal antibody. We also thank Dr. Raul Mena for providing access to confocal microscopy facility. We are in debt to Drs. Arturo Ortega, Daniel Martınez-Fong, Gilda Flores, Dalila Martınez and Victor Aleman for their valuable advice and discussion. This work was partially supported by CON-ACyT, Mexico: Grants 26392-M and 34516-M.

\section{References}

[1] A.H. Ahn, L.M. Kunkel, The structural and functional diversity of dystrophin, Nat. Genet. 3 (1993) 283 - 291.

[2] R.C. Austin, P.L. Howard, V.N. D’Souza, H.J. Klamut, P.N. Ray, Cloning and characterization of alternatively spliced isoforms of Dp71, Hum. Mol. Genet. 4 (1995) $1475-1483$.

[3] R.C. Austin, G.E. Morris, P.L. Howard, H.J. Klamut, P.N. Ray, Expression and synthesis of alternatively spliced variants of Dp71 in adult human brain, Neuromuscular Disord. 10 (2000) 187 - 193.

[4] G. Bai, B. Weiss, The increase of calmodulin in PC12 cells induced by NGF is caused by differential expression of multiple mRNAs for calmodulin, J. Cell. Physiol. 149 (1991) $414-421$.

[5] S. Bar, E. Barnea, Z. Levy, S. Neuman, D. Yaffe, U. Nudel, A novel product of the Duchenne muscular dystrophy gene which greatly differs from the known isoforms in its structure and tissue distribution, Biochem. J. 272 (1990) 557 - 560.

[6] D.J. Blake, R. Hawkes, R. Nawrotzki, N.Y. Loh, D.C. Gorecki, K.E. Davies, hDystrobrevin, a member of the dystrophin-related protein family, Proc. Natl. Acad. Sci. U. S. A. 95 (1998) $241-246$.

[7] D.J. Blake, R. Hawkes, M.A. Benson,P.W. Beesley, Different dystrophin-like complexes are expressed in neurons and glia, J. Cell Biol. 147 (1999) 645 - 658.

[8] G.S. Bloom, R.B. Vallee, Association of microtubule-associated protein 2 (MAP2) with microtubules and intermediate filaments in cultured brain cells, J. Cell Biol. 96 (1983) $1523-1531$.

[9] F.M. Boyce, A.H. Beggs, C. Feener, L.M. Kunkel, Dystrophin is transcribed in brain from a distant upstream promoter, Proc. Natl. Acad. Sci. U. S. A. 88 (1991) $1276-1280$.

[10] T.J. Byers, H.G. Lidov, L.M. Kunkel, An alternative dystrophin transcript specific to peripheral nerve, Nat. Genet. 4 (1993) $77-81$. 
[11] R.G. Ceccarini, M. Cavaldesi, M. Zini, T.C. Petrucci, Localization of the dystrophin binding site at the carboxyl terminus of h-dystroglycan, Biochem. Biophys. Res. Commun. 223 (1996) 272 - 277.

[12] B. Cisneros, A. Rendon,V. Genty,G. Aranda,F. Marquez, D. Mornet, C. Montanez, Expression of dystrophin Dp71 during PC12 cell differentiation, Neurosci. Lett. 213 (1996) $107-110$.

[13] G. Davidkova, S.P. Zhang, R.A. Nichols, B. Weiss, Reduced level of calmodulin in PC12 cells induced by stable expression of calmodulin antisense RNA inhibits cell proliferation and induces neurite outgrowth, Neuroscience 75 (1996) 1003 - 1019.

[14] M.D. Davison, D.R. Critchley, $\alpha$-Actinin and the DMD protein contain spectrin-l ike repeats, Cell 52 (1988) 159 - 160.

[15] V.N. D’Souza, T.M. Nguyen, G.E. Morris, W. Karges, D.A. Pillers, P.N. Ray, A novel dystrophin isoform is required for normal retinal electrophysiology, Hum. Mol. Genet. 4 (1995) $837-842$.

[16] J.M. Ervasti, K.P. Campbell, Membrane organization of the dystrophinglycoprotein complex, Cell 66 (1991) $1121-1131$.

[17] E. Fabbrizio, U. Nudel, G. Hugon, A. Robert, F. Pons, D. Mornet, Characterization and localization of a $77 \mathrm{kDa}$ protein related to the dystrophin gene family, Biochem. J. 29 (1994) 359 - 365.

[18] M. Fukuda,Y. Gotoh,T.Tachibana, K. Dell, S. Hattori,Y.Yoneda, E. Nishida, Induction of neurite outgrowth by MAP kinase in PC12 cells, Oncogene 11 (1995) $239-244$.

[19] E. Gonzalez, C. Montanez, P.N. Ray, P.L. Howard, F. Garcia-Sierra, D. Mornet, B. Cisneros, Alternative splicing regulates the nuclear or cytoplasmic localization of dystrophin Dp71, FEBS Lett. 482 (2000) 209 - 214.

[20] D.C. Gorecki, A.P. Monaco, J.M. Derry, A.P. Walker, E.A. Barnard, P.J. Barnard, Expression of four alternative dystrophin transcripts in brain regions regulated by different promoters, Hum. Mol. Genet. 1 (1992) 505 - 510.

[21] D.C. Gorecki, E.A. Barnard, Specific expression of G-dystrophin (Dp71) in the brain, NeuroReport 6 (1995) 893 - 896.

[22] D.S. Greenberg, Y. Schatz, Z. Levy, P. Pizzo, D. Yaffe, U. Nudel, Reduced levels of dystrophin associated proteins in the brains of mice deficient for Dp71, Hum. Mol. Genet. 5 (1996) 1299 - 1303.

[23] P.W. Gunning, G.E. Landreth, M.A. Bothwell, E.M. Shooter, Differential and synergistic actions of nerve growth factor and cyclic AMP in PC12 cells, J. Cell Biol. 89 (1981) 240- 245.

[24] S. Halegoua, R.C. Armstrong, N.E. Kremer, Dissecting the mode of action of a neuronal growth factor, Curr. Top. Microbiol. Immunol. 165 (1991) 119 - 170.

[25] R.G. Hammonds Jr., Protein sequence of DMD gene is related to actin-binding domain of alpha-actinin, Cell 51 (1987) 1.

[26] E.P. Hoffman, L.M. Kunkel, R.H. Brown Jr., Proteolytic fragment or new gene product?, Nature 336 (1988) 210.

[27] P.L. Howard, H.J. Klamut, P.N. Ray, Identification of a novel actin binding site within the Dp71 dystrophin isoform, FEBS Lett. 441 (1998) 337 - 341.

[28] J.P. Hugnot, H. Gilgenkrantz, N. Vincent, P. Chafey, G.E. Morris, A.P. Monaco, Y. Berwald-Netter, A. Koulakoff, J.C. Kaplan, A. Kahn, Distal transcript of the dystrophin gene initiated from an alternative first exon and encoding a 75-kDa protein widely distributed in nonmuscle tissues, Proc. Natl. Acad. Sci. U. S. A. 89 (1992) $7506-7510$. 
[29] V. Jancsik, B. Gerics,F. Hajos, B. Jenei, D. Filliol, A. Rendon, New polyclonal antiserum against microtubule-associated Protein 2 (MAP2); preparation and preliminary characterization, Neurobiology 4 (1996) 241 - 245.

[30] D. Jung, D. Fillol, M.H. Metz-Boutigue, A. Rendon, Characterization and subcellular localization of the dystrophin-protein 71 (Dp71) from brain, Neuromuscular Disord. 3 (1993) 515 - 518.

[31] H.G. Lidov, S. Selig, L.M. Kunkel, Dp140: a novel 140 kDa CNS transcript from the dystrophin locus, Hum. Mol. Genet. 4 (1995) 329 - 335.

[32] N.Y. Loh, S.E. Newey, K.E. Davies, D.J. Blake, Assembly of multiple dystrobrevin-containing complexes in the kidney, J. Cell Sci. 113 (2000) 2715 2724.

[33] N.M. Maraldi, G. Lattanzi, P. Sabatelli, A. Ognibene, S. Squarzoni, Functional domains of the nucleus: implications for Emery-Dreifuss muscular dystrophy, Neuromuscular Disord. 12 (2002) 815 - 823.

[34] F. Marquez, et al., Differential expression and subcellular distribution of dystrophin Dp71 isoforms during differentiation process, Neuroscience 118 (2003) $957-966$.

[35] M.P. Moizard, A. Toutain, D. Fournier, F. Berret, M. Raynaud, C. Billard, C. Andres, C. Moraine, Severe cognitive impairment in DMD: obvious clinical indication for Dp71 isoform point mutation screening, Eur. J. Hum. Genet. 8 (2000) $552-556$.

[36] A.C. Murnane, K. Brown, C.H. Keith, Preferential initiation of PC12 neurites in directions of changing substrate adhesivity, J. Neurosci. Res. 67 (2002) 321 - 328.

[37] F. Pons,N. Augier,J. Leger,A. Robert,F.S.M.Tome,M. Fardeau,T. Voit, L.V.B.D. Nicholson, J.J. Leger, A homologue of dystrophin is expressed at the neuromuscular junctions of normal individuals and DMD patients and of normal and mdx mice: immunological evidence, FEBS Lett. 282 (1991) 161 - 165.

[38] A. Renaudin, M. Lehmann, J. Girault, L. McKerracher, Organization of point contacts in neuronal growth cones, J. Neurosci. Res. 55 (1999) $458-471$.

[39] H. Rosas-Vargas, C. Montanez, A. Rendon, D. Mornet, F. Garcia, V. Ceja, B. Cisneros, Expression and localization of utrophin in differentiating PC12 cells, NeuroReport 11 (2000) 2253 - 2257.

[40] R. Sarig, V. Mezger-Lallemand, I. Gitelman, C. Davis, O. Fuchs, D. Yaffe, U. Nudel, Targeted inactivation of Dp71, the major non-muscle product of the DMD gene: differential activity of the Dp71 promoter during development, Hum. Mol. Genet. 8 (1999) 1 - 10.

[41] A. Suzuki, M.Yoshida, H.Yamamoto, E. Ozawa, Glycoprotein-binding site of dystrophin is confined to the cysteine-rich domain and the first half of the carboxyterminal domain, FEBS Lett. 308 (1992) $154-160$.

[42] R.R.Vaillancourt, A.M. Gardner, G.L. Johnson, B-raf-dependent regulation of the MEK-1/mitogen-activated protein kinase pathway in PC12 cells and regulation by cyclic AMP, Mol. Cell. Biol. 14 (1994) 6522 - 6530.

[43] D.M. Vogt Weisenhorn, L.J. Roback, J.H. Kwon, B.H. Wainer, Coupling of cAMP/PKA and MAPK signaling in neuronal cells is dependent on developmental stage, Exp. Neurol. 169 (2001) 44- 55.

[44] M. Yoshida, E. Ozawa, Glycoprotein complex anchoring dystrophin to sarcolemma, J. Biochem. (Tokyo) 108 (1990) 748 - 752. 